INTERNATIONAL JOURNAL OF RESEARCHES IN BIOSCIENCES, AGRICULTURE AND TECHNOLOGY

(C) VISHWASHANTI MULTIPURPOSE SOCIETY (Global Peace Multipurpose Society) R. No. MH-659/13(N) www.vmsindia.org

\title{
ELECTRICAL CONDUCTIVITY OF CHEMICALLY SYNTHESIZED POLYANILINE FLY ASH COMPOSITES
}

\author{
A. D. Dahegaonkar 1 , S. B. Kondawar ${ }^{2}$ and V. A. Thabhane ${ }^{3}$ \\ ${ }^{1}$ N.S. Science and Arts College, Bhadrawati, Dist. Chandrapur \\ ${ }^{2}$ Department of Physics, R. T. M. Nagpur University, Nagpur \\ ${ }^{3}$ Department of Physics, Pune University, Pune \\ ajaydahegaonkar@gmail.com
}

\begin{abstract}
In the past few decades, Fly Ash (FA) has rapidly emerged as one of the most cost-effective and environment friendly resources rather than just being a waste material. Most of the current applications of FA, however, are primarily based on its microstructure and chemical composition only. Other physical and chemical properties of FA, and of the materials based on it, could possibly be utilized in other practical applications also. With this idea, we report in this paper about the synthesis, characterization and electric conductivity of a composite made of FA together with a very common conducting polymer Polyaniline (PANI). The polyaniline/fly ash (PANIFA) composites with various concentrations $(10,20,30.40$ and $50 \% w t)$ of fly ash were synthesized by the process of in-situ polymerization by chemical oxidization method. Powder X-ray diffraction (XRD), scanning electron microscopy (SEM) and UV-Vis spectroscopy were used to characterize the samples. The d c-conductivity of the samples was measured as a function of temperature in the range $30^{\circ} \mathrm{C}-150^{\circ} \mathrm{C}$. An expected decrease of conductivity was observed with addition of FA into PANI. The temperature dependence d c-conductivity for pure PANI and PANIFA composites conclude that electrical properties FA can be used to control the conductivity of PANI to make it suitable for various applications

Keywords: Conducting polymers, Polyaniline, Fly ash, Composites, Electrical conductivity
\end{abstract}

\section{Introduction:}

Polyaniline (PANI) continues to attract considerable attention because its electrical and optical properties can be changed by oxidation and protonation of the amine nitrogen atoms. PANI is known for its excellent thermal and environmental stability but poor processibility due to insolubility and brittleness that limits its commercial applications. PANI could be more processable in the composites form with another water soluble polymers such as PVA, poly (vinyl pyrrolidone), poly (acrylic acid) and poly (styrene sulfonic acid) (PSSA) which are used as stabilisers. A functionalised protonic acid can be added into the composites to chemically polymerise PANI. The PANI dispersion can then be casted to form composite film containing PANI nanoparticles [1]. To improve the conductivity further, conducting polymers have been irradiated with x-rays [2, 3], gamma radiation [4], and gamma and electron beams [5]. When ionising radiation interacts with polymer materials active species such as free radicals are produced, thereby initiating chemical reactions including cross linking, chain scission, and grafting. The ionic carriers induced by radiation scission can improve the conductivity of PANI. The conducting polyaniline (PANI) is one of the promising conducting polymers due to its high conductivity, ease preparation, good environmental stability and large variety of applications [6] which make this polymer suitable for gas sensor, functional hybrid, as $\mathrm{pH}$ swchiting electrical conducting biopolymer hybrid for sensor applications, as an electrically active redox biomaterial for sensor applications, as a matrix for preparation of conducting polymer nanocomposites [7]. Therefore, there has been increasing interest of the researchers for the preparation of nanocomposites based on PANI. The literature review on conducting polymer nanocomposites show that PANI has been successfully utilized for the preparation of nanocomposites [8,9]. Various applications of conducting polymers have been proposed as transducers of biosensor [10], electrodes of rechargeable batteries, artificial nerves and muscles, gas sensors, solid electrolytic capacitor, diodes and transistors, anti-static electromagnetic shielding, and biomedical applications.

Coal-based thermal power stations have been operated for more than 50 years in India, but the concept of developing environmentfriendly solutions for fly ash utilization is major concern now a day. Overall fly ash utilization in India stands at a fairly low level of about $15 \%$ of the quantity generated. Several factors to be considered for fly ash utilization in India, among numerous factors that account for the low level of utilization, are listed as follows: 1) Poor understanding of the chemistry of fly ash and its derivatives for proper end applications. 2) Absence of standards and specifications for fly ash products. 3) Lack of reliable quality assurance for fly ash products Poor public awareness about the products and their performance. 4) Non-availability of dry fly ash collection facilities Easy availability of land with 
top soil at cheap rates for manufacturing conventional bricks. 5) Lack of proper coordination between thermal plants and ash users. Fly ash utilization in the country is gaining momentum owing to the stringent regulations that the Ministry of Environment and Forests, Government of India has stipulated, as also to increased awareness about the benefits of using fly ash for various products [11].

\section{Experimental}

The GR grade Aniline was purified by distillation under reduced pressure. A fine fresh clean and pure FA powder was collected from the Thermal Power Station, Chandrapur, India. FA contains elements like $\mathrm{Cu}, \mathrm{Pb}, \mathrm{Cd}, \mathrm{Ag}, \mathrm{Mn}$, $\mathrm{Ti}, \mathrm{Na}, \mathrm{Mo}, \mathrm{S}, \mathrm{P}, \mathrm{Zn}$ and $\mathrm{Cl}$ in different concentrations [12]. $0.4 \mathrm{M}$ of distilled Aniline was added to the solution of $0.4 \mathrm{M}$ of ammoniumpersulfate $\left[\left(\mathrm{NH}_{4}\right)_{2} \mathrm{~S}_{2} \mathrm{O}_{8}\right]$ and this reaction mixture was stirred continuously at room temperature to obtain polyaniline. To this reaction mixture, varied weight per cent of fly ash powder (10 20,30, 40 and 50) was added to form polyaniline-fly ash composites. The obtained product was filtered and washed thoroughly with methanol $\left(\mathrm{CH}_{3} \mathrm{OH}\right)$ and the sample was dried under vacuum for more than $24 \mathrm{~h}$ at room temperature. The obtained composites were pressed in the form of circular pellets of $1 \mathrm{~cm}$ diameter. The SEM images of polyaniline-fly ash composites were investigated using Field Emission Gun Scanning Electron Microscope. The X-ray diffraction patterns of the samples in this present case were recorded on Philips PW-1700 X-ray diffractometer using CuKa radiation of wavelength $1.544 \mathrm{~A}^{0}$ Continuous scan of $2 \%$ minimum with accuracy of 0.01 . The characterization of polyaniline and its composites by spectroscopic methods is important, as it gives information not only about various molecular-level interactions but also on the type of charge carriers.

\section{Results and Discussion:}

$U V$-Visible spectroscopy

The UV-Vis absorption spectra of PANI and PANIFA composite are shown in Fig. 1. Two absorption bands are observed in the wavelength region from 315 to $350 \mathrm{~nm}$ and a small band at 578 to $712 \mathrm{~nm}$ for the PANI. PANI always exhibits a $\Pi-\Pi^{*}$ transition, usually closer to $315 \mathrm{~nm}$ [13]. Partially oxidized PANI and its oligomers display an additional absorption at around $712 \mathrm{~nm}$ associated with the quinoid (oxidized) units [14]. These peaks are characteristic of the PANI emeraldine base and indicate that nanostructure PANI composites are stabilized in the emeraldine base redox state.

The peak at $315 \mathrm{~nm}$ is attributed to $\pi-$ $\Pi^{*}$ transition of benzoid rings and the peak at $712 \mathrm{~nm}$ is attributed to the charge transfer excitation of the quinoid structure. PANIFA prepared without aging show clear similarity in their UV-Vis spectra particularly with the complete absence of the absorption maxima at 320 and $630 \mathrm{~nm}$ which is associated with the stabilization of the composite in the emeraldine form, comparatively PANIFA composite show clear similarity in their UV-Vis spectra particularly with the presence of the absorption maxima at 315 and $610 \mathrm{~nm}$ which is associated with the stabilization of the composite in the emeraldine form. Comparison of the PANI and PANIFA composite spectra shows that FA stabilizes the polyanilines in its emeraldine form [15].

\section{Scanning Electron Microscopy}

Fig. 2 shows the SEM micrograph of PANI, FA and PANIFA composites. SEM of PANI shows porous, non-uniform structure. SEM is showing the general features of the original fly ash. As it can be seen in the figure, the fly ash is mainly constituted by compact or hollowed spheres but with a regular smooth texture. Also, some quartz particles, residue of un-burnt coal or some vitreous unshaped fragments could be seen. A very high magnification reveals the homogeneous distribution of fly ash (cenosphere) particles. PANIFA composite shows the formation of dine base form of PANI significantly changes the aggregate state of polymeric molecular chain. It is seen from the micrograph that cluster and granular structure of polyaniline is maintained even after the addition of fly ash in polyaniline. Hence, a network of fly ash and granular polyaniline has been formed in case of composites.

\section{$X$ - ray diffraction}

Fig. 3 shows the XRD of pure PANI and PANIFA composites. It has been suggested by XRD study that PANI undergoes interfacial interaction with FA crystallites and loses its own morphology by its mixing with fly ash. PANIFA composites show peaks of fly ash as well as polyaniline indicating that fly ash crystallites have been uniformly mixed within the polymer chain.. Careful analysis of X-ray diffractogram of polyaniline-fly ash composite suggests that it exhibits semicrystalline behavior. No structural change has been observed in fly ash due to its dispersion in polymerization of reaction of polyaniline [16].

\section{Conductivity measurements:}


To study the conduction mechanism of fly ash in pellet form, the DC electrical conductivity was determined by measuring current and voltage in four probe resistivity set up at different temperatures. Fig. 4 shows the variation of conductivity with temperature for the fly ash. Fig, 4 shows the variation of dc conductivity ( $\sigma$ dc) with the temperature for pure PANI and fly ash filled PANI composites. The dc conductivity of PANI decreases by the addition of fly ash. In polymers conduction takes place by the mobility of charge carriers from an occupied site to an unoccupied site. The defects generally increase with temperature and are independent of temperature at lower temperatures. At higher temperatures additional defects are created. At lower temperatures, they are frozen. Hence, the conductivity variation with temperature is divided into two regions: (I) pertaining to higher temperature and (II) pertaining to lower temperature [17]. It may be easily noted that the conductivity decreases with the increasing concentrations of FA. The room temperature resistivity increases (conductivity decreases) with the addition of FA in PANI. [18].From the study of dc conductivity as a function of temperature, it was inferred that both the PANIFA composites as well as the pure PANI were semiconducting in nature. Moreover, the conductivity was expectedly found to decrease with the addition of FA in PANI [19].

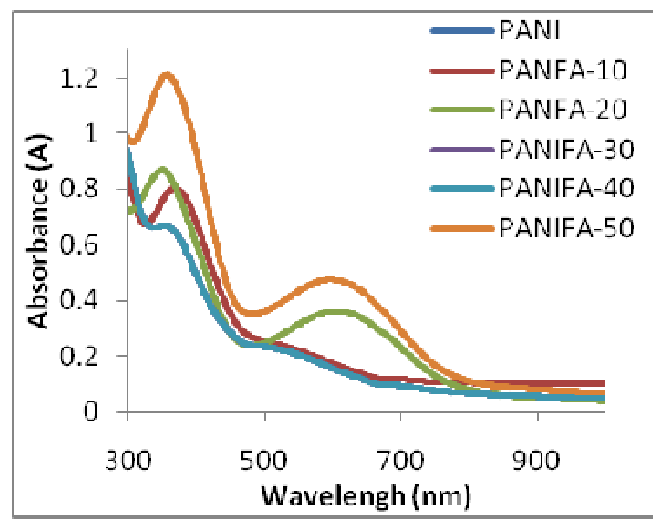

Figure. 1- UV-Vis spectra of PANIFA Composites
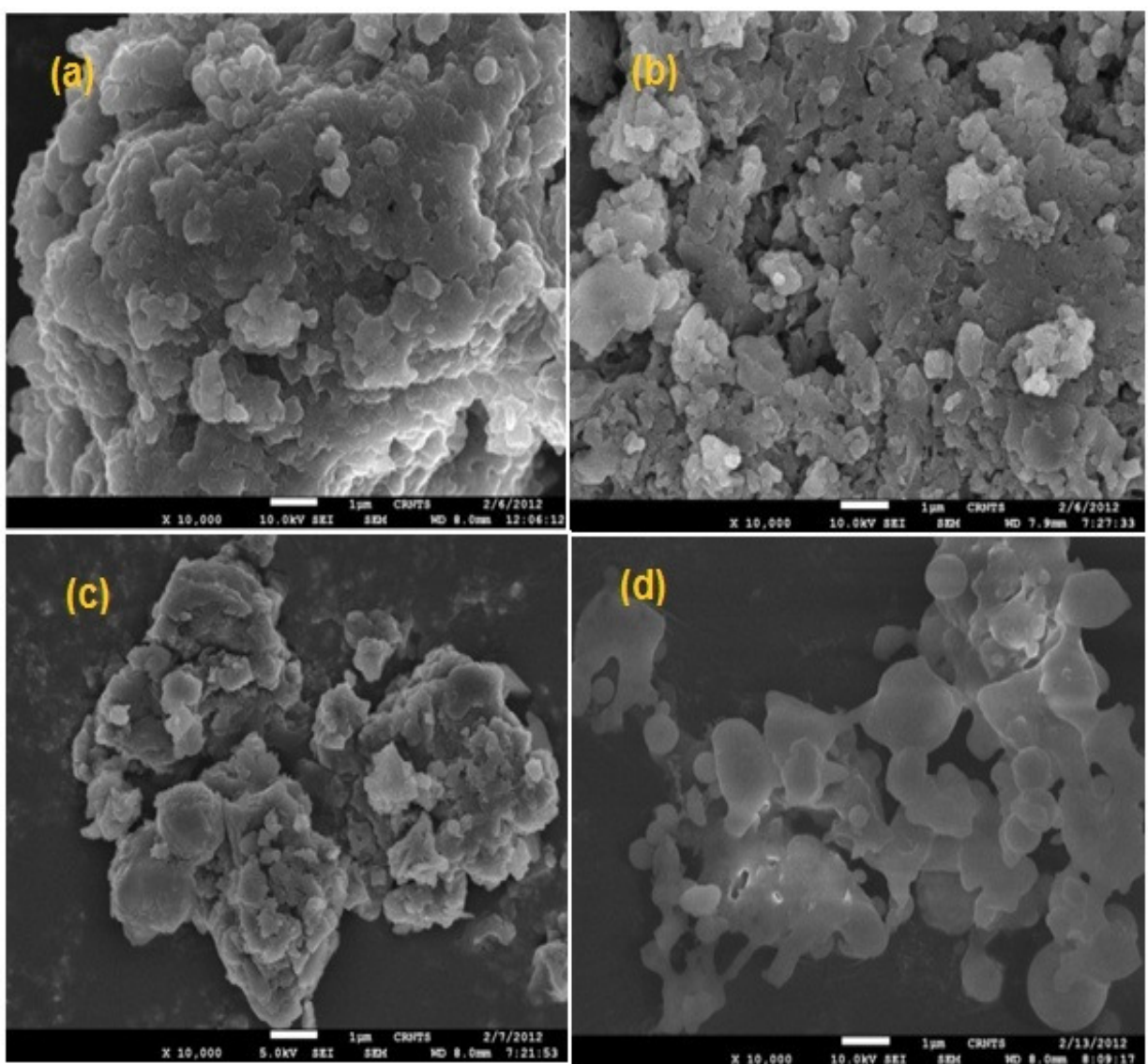

Figure. 2: Scanning electron micrograph of (a) PANI, (b) PANIFA-10, (c) PANIFA-40, (d) FA 


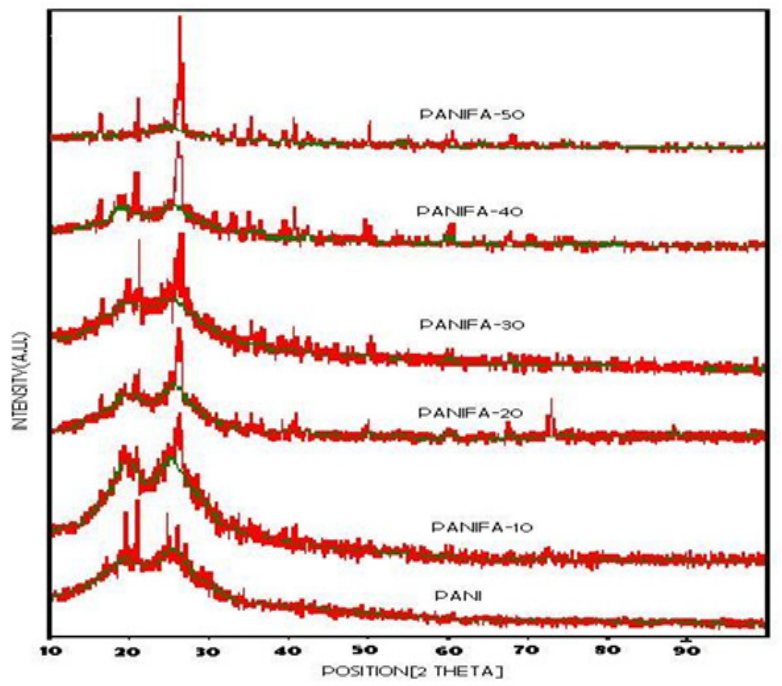

Figure. 3: X-ray diffraction pattern of PANI, PANIFA Composites.

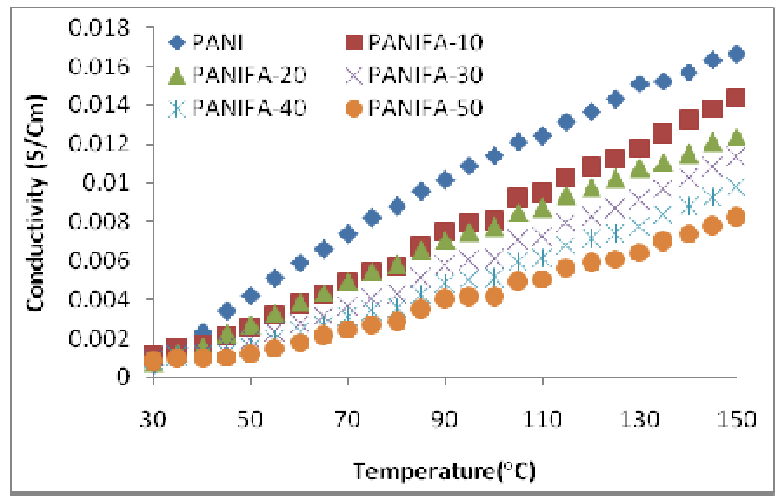

Figure. 4- Electrical conductivity of PANIFA Composites

\section{Conclusion:}

With more than 100 million tones of fly ash produced in India, use of fly ash for the preparation of polyaniline- fly ash composites will in no way help in its bulk utilization. Still the authors have made an effort towards the better utility of fly ash by synthesizing polyaniline -fly ash composites. The detailed characterization of these composites was successfully carried out through XRD, SEM and UV-vis. Electrical property is an important property of material. The results of electrical show a strong dependence on the weight percent of fly ash in polyaniline which can be best suitable electronics application.

\section{Acknowledgement:}

Dr. A. D. Dahegaonkar is thankful to University Grants Commission, Western Region Office, Pune (India) for financial support to carry out this research work under minor research project UGC F. No. 47-1337/ 10 (WRO).

\section{References:}

[1] C M. Seong, S.Y., Park, , J.Y. Hwang \& H.J. Choi, "Synthesis and electrical properties of polymer composites with polyaniline
nanoparticles”.Mater.Sci. Eng C (24), 15 -18, 2004

[2] J.A. Malmonge,\&, L.H.C. Mattoso “ Doping of polyaniline and derivatives induced by X-ray radiation" Synthetic Metals.84, 779-780, 1997

[3] A. Dhanabalan, J. A. Malmonge, Jr, A Riul., R.M. Faria, \& Jr, O.N. Olivera "A study on X-ray irradiation of composite polyaniline LB films" Thin Solid Films.327-29 (22), 808-812, 1998

[4] H. Bodugoz, U.A. Sevil \& O. Guven, "Radiation-induced conductance in the blends of poly(aniline-base) with poly(vinyl Chloride) and poly(vinylidene chloride)-co-(vinyl acetate)" Mcromol. Symp. 169,289-295, 1998

[5] U.A. Sevil, O.Güven, , A. Kovács, \& I. Slezsák, "Gamma and electron dose response of the electrical conductivity of polyaniline based polymer composites" Radiation Physics and Chemistry. 67, 575-580, 2003

[6] S.R Kargirwar S.R Thakare, M.D. Choudhary, S.B.Kondawar, S.R Dhakate.; "Morphology And Electrical Conductivity Of Selfdoping Polyanilines Synthesized Via Selfassembly Process" Adv Mat Lett. 2(6), 397-400, 2011

[7] S.B.Kondawar; S.R Thakare,. S. Bompilwar,V Khati " Nanostructure Titania Reinforced Conducting Polymer Composites" Int. J. Mod. Phys. B, 23 (15), 3297 -3304, 2009

[8] S.B. Kondawar, S.A Acharya,. S.R.Dhakate, Adv. Mat. Lett.2 (5),362.-367 "Microwave assisted hydrothermally synthesized nanostructure zinc oxide reinforced polyaniline nanocomposites," Advanced Materials Letters, 2(5), 362-367, 2011.

[9] A. K. Sharma,Y. Sharma, R.; Malhotra J.K Sharma, "Solvent Tuned PANI-CNT composites as advanced electrode materials for supercapacitor application" Adv.Mat. Lett 3(2), 82-86, 2012

[10] A Kros,V. Hovel, S. W. Nolte , R.J.M N Sommerdijk, "A printable glucose sensor based on a poly(pyrrole)-latex hybrid material" Sensors and Actuators. 80, 229-233, 2001

[11] S. V. Patil, S.C. Nawle ,S. J. Kulkarni “ Industrial Applications of Fly ash: A Review" International Journal of Science, Engineering and Technology Research (IJSETR), 2(9), 2013

[12] A. Volgel "A text book of quantitative inorganic analysis", second edition, (London: Longman), 1969

[13] Y.Cao, S. Z. Li, Z. J. Xuea and D. Guo, "Spectroscopic and Electrical Characterization of 
Some Aniline Oligomers and Polyaniline," Synthetic Metats, Vol. 16, No. 3, 305-315, 1986,

[14] J.Libert, J. Cornil, D. A. dos Santos and J. L. Bredas, "A Theoretical Investigation of from Neutral Oligoanilines to Polyanilines" The Chain-Length Dependence of the Electronic and Optical Properties” Physical Review B, 56, (14), 8638-8650, 1997

[15] R. Khan,P. Khare,B. Prasad,A. Kumar H.N. Dey. "Spectroscopic, Kinetic Studies of Polyaniline-Flyash Composite" Advances in Chemical Engineering and Sciences 1, 37-44, 2011

[16] S.B. Kondawar, S.A Acharya,. S.R.Dhakate, "Microwave assisted hydrothermally synthesized nanostructure zinc oxide reinforced polyaniline nanocomposites" Adv. Mat.Lett.2 (5), 362-367, 2011
[17] V. Chand, M. Mehra, "Thermal and dc Conductivity Behavior of Fly Ash Filled Polyaniline Composites" Polymer Plastics Technology and Engineering, 47(9), 883-886, 2008

[18] H. Narayan, H. Alemu, and E. Iwuoha phys. stat. sol. (a) 203(15), 3665-3672,2006

[19] H. Narayan, H. Alemu, V. S. Somerset, E. I. Iwuoha, M. L. Hernández, A. Hernández, A. M. Montaño and J. A. Henao In book: "Fly Ash: Reuse, Environmental Problems, and Exposure Issues," Chapter: 5, "Fly Ash Composites with Polyaniline: Synthesis, Characterization and Conductivity Measurements" Publisher: Nova Science. Publishers, New York, 111-136, 2009 